\title{
Best Practice Guidelines for Breast Imaging, Breast Imaging Society, India: Part-1
}

\author{
Suma Chakrabarthi ${ }^{1}$ Shikha Panwar ${ }^{2}$ Tulika Singh $^{3} \quad$ Shilpa Lad $^{4}$ Jwala Srikala ${ }^{5}$ \\ Niranjan Khandelwal ${ }^{6}$ Sanjeev Misra ${ }^{7}$ Sanjay Thulkar $^{8}$
}

${ }^{1}$ Department of Radiology and Imaging, Peerless Hospitex Hospital and Research Center Limited, Kolkata, West Bengal, India

${ }^{2}$ Department of Radiology, Mahajan Imaging, Delhi, India

${ }^{3}$ Department of Radiodiagnosis and Imaging, Postgraduate Institute of Medical Education and Research, Chandigarh, India

${ }^{4}$ Department of Radiology, NM Medical, Mumbai, Maharashtra, India

${ }^{5}$ Department of Radiology and Imaging, Krishna Institute of Medical Sciences, Secunderabad, Telangana, India

6 Former Head, Department of Radiodiagnosis and Imaging, Postgraduate Institute of Medical Education and Research, Chandigarh, India

${ }^{7}$ Department of Surgical Oncology, All India Institute of Medical

Sciences, Jodhpur, Rajasthan, India

${ }^{8}$ Department of Radiology, Dr. B.R. Ambedkar Institute Rotary Cancer

Hospital, All India Institute of Medical Sciences, New Delhi, India
Address for correspondence Suma Chakrabarthi, MBBS, MRCS, FRCR, Department of Radiology and Imaging, Peerless Hospitex Hospital and Research Center Limited, 360, Pancha Sayar, Kolkata, West Bengal 700094, India (e-mail: sumadoc@gmail.com).

Ann Natl Acad Med Sci (India) 2022;58:60-68.

\author{
Abstract

\section{Keywords} \\ - Indian breast imaging \\ guidelines \\ - best practice \\ guidelines for breast \\ imaging \\ - mammography \\ - breast ultrasound \\ - breast MRI \\ - breast symptoms
}

Breast imaging is one of the prerequisites for providing high quality breast health care. Choosing the appropriate investigation is central to diagnosing breast disease in women and men who present to health professionals for treatment. Patients with breast disease present to doctors of different subspecialties as well as general practitioners in our country. It is important therefore to provide uniform guidance to doctors in different health care setups of our country, urban and rural, government and private, for breast diseases to be diagnosed and treated optimally. These guidelines framed by the task group set up by the Breast Imaging Society, India have been formulated focusing primarily on the Indian patients and health care infrastructures. These guidelines aim to provide a framework for the referring doctors and practicing radiologists, to enable them to choose the appropriate investigation for patients with breast symptoms and signs. The aim has been to keep this framework simple and practical so that it can guide not only subspecialists in breast care but also help doctors who do not routinely deal with breast diseases, so that breast cancer is not missed. Overall, the aim of this document is to provide a holistic approach to standardize breast care imaging services in India. Part 1 of these guidelines focuses on the best practice principles for mammography, breast ultrasound and breast magnetic resonance imaging. In the absence of a populationbased screening program in India, the guidelines to be followed for those women who wish to be screened by mammography have been provided. The key points of these guidelines include the recommendations that mammography is the modality of choice for breast published online

February 16, 2022
DOI https://doi.org/

$10.1055 / \mathrm{s}-0042-1742586$. ISSN $0379-038 X$.

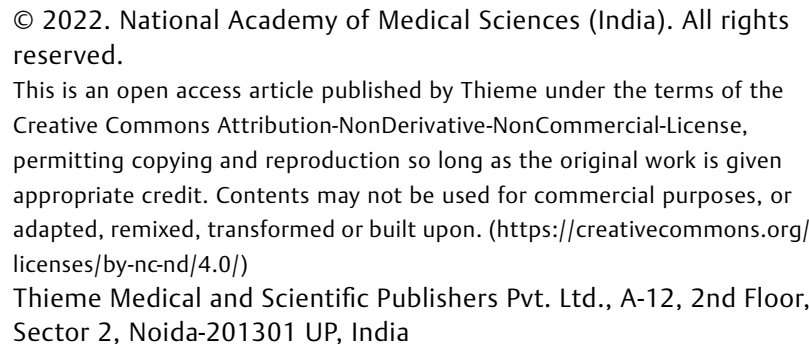

(c) 2022. National Academy of Medical Sciences (India). All rights reserved.

This is an open access article published by Thieme under the terms of the Creative Commons Attribution-NonDerivative-NonCommercial-License, permitting copying and reproduction so long as the original work is given appropriate credit. Contents may not be used for commercial purposes, or adapted, remixed, transformed or built upon. (https://creativecommons.org/ licenses/by-nc-nd/4.0/)

Thieme Medical and Scientific Publishers Pvt. Ltd., A-12, 2nd Floor, Sector 2, Noida-201301 UP, India 
screening and investigation of symptomatic women aged over forty years. Screening is advised annually from the age of forty. Ultrasound is the investigation of choice for pregnant and lactating women and women less than thirty years of age. For women between thirty to thirty-nine years of age, ultrasound can be used initially followed by mammography in presence of clinical or sonographic suspicion of breast cancer. All women diagnosed with breast cancer should have ultrasound and mammography. Breast MRI is useful for assessment of disease extent, problem solving, evaluation of response to neo-adjuvant chemotherapy, identifying occult breast primary and evaluation of augmented breasts.

\section{Mammography}

\section{Introduction}

Mammography has stood the test of time and is considered the best screening tool for breast cancer. It is the first line of investigation for most breast symptoms. As an investigative tool, it helps characterize breast lesions and thus plays a significant role in reducing mortality and morbidity from breast cancer. Along with ultrasound, it continues to be the basic investigative tool for breast diseases. Even when breast screening magnetic resonance imaging (MRI) study is performed, it is very useful to have a correlating mammogram available. As a breast radiologist, it is therefore most important to master the art and technique of performing and reporting mammography. In this document, indications and technique of mammography have been discussed. Available international data as well as Indian scenario and need have been taken into consideration and breast screening guidelines from the Breast Imaging Society, India (BISI) have been recommended in this document.

\section{Technique}

Establishing a rapport with the client and explaining the procedure helps alleviate client's anxiety. This goes a long way in acquiring images that are optimal and useful.

Mammography should include mediolateral oblique (MLO) and craniocaudal (CC) views of each breast. These are regarded as the standard views. Even when symptoms are unilateral, bilateral mammograms are advised to assess asymmetric abnormalities. Digital mammography is preferred to film screen mammography, particularly for women aged younger than 50 years and for those with dense breast tissue. ${ }^{1}$ If a suspicious abnormality is demonstrated on mammography, it is helpful to further characterize the mammographic features using magnification or spot compression views. Other views such as the laterally and medially extended CC views and the valley view help visualize areas of the breast that are difficult to visualize on the standard views. Lateral view helps confirm if a lesion seen on the MLO view is truly in the upper or lower half of the breast.

Adequate quality assurance of the mammography unit is mandatory. The technique of acquisition of mammographic images must be monitored with specific attention to radiation dose and technical adequacy of films. The dose of radiation must be minimized based on the As Low As Reasonably Achievable principle. Most mammography units have an automated exposure control system, which helps minimizing radiation for the given breast thickness and composition. Exposure time must be as low as possible, to reduce dose, to avoid motion artifact, and to minimize discomfort to the woman being imaged. Optimal compression must be applied.

\section{Indication}

Broadly, the indications for mammography fall into two categories: screening and diagnostic.

Screening mammograms: These are for women who have no breast symptoms and no clinical signs of breast cancer. The purpose is early detection of breast cancer, when it is small and impalpable. This is to aid reduction of patient morbidity and mortality.

Diagnostic mammograms: These are for women and men who have a symptom such as a palpable lump or bloodstained nipple discharge. The mammograms are acquired to identify the cause of the symptom, more specifically to diagnose if the symptoms are caused by a malignant mass.

\section{Screening Mammograms}

The population-based cancer registries of India have shown a significant increase in number of breast cancers diagnosed over the years in India. ${ }^{2}$ It has been felt by different groups that breast self-examination and clinical breast examination (CBE) are perhaps the right tools for early detection of breast cancer for the huge population of India. Mammography has established itself as the investigation of choice for breast screening. Breast screening-related information, both potential benefits and possible risks, must be given to the woman and mammography must be performed after the woman gives an informed consent.

There are many screening guidelines from countries that have population-based breast screening programs. These programs vary from country to country and hence there are different guidelines from different breast societies in the world. Due to the lack of data specific to our country, currently, no specific guidelines are available in India. However, a few concepts and facts are recognized all over the world, and these can be applied to the Indian population in general. There are other facts that are specific to our country, such as increasing incidence of breast cancer in younger women, and the practice in India needs to be planned keeping these in mind. Some examples of breast screening program/guidelines are the National Health Service Breast Screening Program $(\mathrm{UK})^{3}$ and the American College of Radiology guidelines. ${ }^{4}$ 
For women at average risk of breast cancer, screening mammography is thought to be beneficial between the ages of 50 and 74 years. ${ }^{5}$ Although mortality reduction is less than that seen when screening older women, randomized controlled trials have shown a significant reduction in mortality in the 40 to 49 years age group. ${ }^{6}$ Mammography screenings are effective and generate a $17 \%$ reduction in breast cancer mortality in women 39 to 49 years of age. ${ }^{7}$ Breast cancer incidence increases with age. As a result, more women in the 40 to 50 years age group would need to be screened to save the same number of lives as would be saved by screening women $\geq 50$ years of age. But due to the longer life expectancy in younger women, life years gained for the women diagnosed with breast cancer by screening in their 40 s is higher than in the $\geq 50$ year old age group. ${ }^{8}$ It is known that screening women aged 40 to 49 years requires more frequent mammography and is less specific than screening in older women. Screening women 40 to 49 years of age does not, however, increase overdiagnosis compared with women starting screening at 50 years of age. ${ }^{6}$ There is no evidence to support screening of women younger than 40 years who are at average risk from breast cancer. ${ }^{5,6}$ No upper age limit is established for screening mammography. However, as the benefits of breast screening may take years to be fully realized, life expectancy and comorbid conditions should be taken into consideration to decide the upper age limit. In general, screening mammography can be considered appropriate when a woman's life expectancy exceeds 5 to 7 years. ${ }^{8}$

Patients presenting with breast cancer are about one decade younger in developing countries in comparison to women in developed countries. ${ }^{9}$ The proportions of young patients ( $<35$ years) of breast cancer vary from $\sim 10 \%$ in developed nations to up to $25 \%$ in developing Asian countries. ${ }^{9}$ In the developing countries, locally advanced cancers constitute more than $50 \%$ of all patients managed indicating that diagnosis happens at a relatively late stage due to multiple causes. ${ }^{9}$ The life expectancy at birth in 2013 to 2017 has been 70.4 years for the female population in India. $^{10}$

\section{Breast Screening Recommendation from Breast Imaging Society, India}

Taking all of the above data into consideration, 40 years is recommended as the age for starting mammography-based screening in India. Although no dedicated population-based screening program exists in India, opportunistic screening of interested women, on a yearly basis, from the age of 40 years, is deemed appropriate. Annual mammography is advised till the age of 70 years. Beyond the age of 70 years, it is advised that a decision is made based on the woman's comorbidities and life expectancy.

Screening (surveillance) of contralateral breast to look for metachronous breast cancer after unilateral mastectomy for breast cancer is to be performed under the age of 40 years, if cancer was detected at $<40$ years of age. Ultrasound and MRI may be required as adjuncts as younger women may have relatively dense breasts.
High-risk group screening (mammograms along with breast MRI) is appropriate if there is a lifetime risk of breast cancer of $\geq$ $20 \%$ according to risk assessment tools that are mainly based on family history, if woman has a known BRCA1 or BRCA2 gene mutation or has a first-degree relative with BRCA1 or BRCA2 gene mutation (and has not had genetic testing herself), if woman has a first-degree relative with premenopausal breast cancer, if she has had radiation therapy to the chest when she was between the ages of 10 and 30 years. ${ }^{4}$ Screening with annual mammography (and annual MRI) is recommended to begin at the age of 30 years or 10 years before the age of diagnosis of firstdegree relative with breast cancer, whichever is later. With history of mantle radiotherapy, annual mammogram (and annual MRI) should be started 8 years after radiation therapy. However, screening mammography is not to be started for any woman before the age of 25 years, irrespective of the cause of high risk. Mammography and MRI are complementary examinations, and both should be performed. ${ }^{4,8}$

\section{Diagnostic Mammograms}

In the presence of breast symptoms such as a palpable lump, blood stained or serous nipple discharge, breast pain that requires investigation, mammography is used in the investigation of women aged 30 years or older. Addition of ultrasound is useful as these two modalities complement each other. In the 30 to 39 years age group, clinical correlation is advised before requesting/performing a mammogram. For example, a 32-year-old woman with a clinically palpable small mobile mass with ultrasound features of a typical fibroadenoma may not require a mammogram for further characterization of the lump. Mammography should not be used for opportunistic screening in the 30 to 39 years age group for women with average risk of breast cancer.

Mammography is not indicated as the first investigation for the majority of patients aged $<30$ years. Ultrasound is the imaging method of choice for the majority of women aged $<30$ years and during pregnancy and lactation. However, mammography should be performed in all patients with proven malignancy. Similarly, mammography should be performed if there is a worrying appearance on ultrasound or clinical examination even in the $<30$ years age group.

Breast pain that needs investigation such as focal persistent noncyclical pain is often a nonspecific breast symptom. In symptomatic women younger than 30 years, ultrasound is more accurate in making a diagnosis than mammography, ${ }^{11}$ and hence ultrasound is recommended as the first investigation. In the 30 to 39 years age group, adding mammography is advised. ${ }^{11}$ Mammography may also be indicated in patients under the age of 30 years if a suspicious lesion is found on the initial ultrasound examination, or if clinical signs justify the radiation exposure. Above the age of 40 years, mammography is recommended as the first investigation. Ultrasound is also useful in this age group as an adjunct to identify the cause of pain, especially in dense breasts.

In women presenting with a lump, diagnostic mammography is recommended if age of the woman is 30 years or older. In the 30 to 39 years age group, clinical correlation is 
advised before requesting/performing a mammogram. Ultrasonography is more sensitive than mammography in detecting lesions in women with dense breasts, and it is the preferred imaging modality in women younger than 30 years with a palpable breast mass. ${ }^{12}$ If ultrasound identifies a suspicious lesion, mammography is advised for the under 30 years age group also.

There are other guidelines which advise a cutoff age of 40 or 35 years for performing mammography in women presenting with breast symptoms. ${ }^{13}$ Given the early onset of breast cancer in India, and given that the Indian data we have are mostly from symptomatic women (rather than cancer picked up at breast screening), a lower cutoff age of 30 years has been deemed appropriate for women with symptoms in our country.

Please read the guidelines on "Common Breast Symptoms: Algorithm for Imaging Evaluation," which is a section of these best practice guidelines for further information on the appropriate tests for patients presenting with breast symptoms.

\section{Reporting Mammograms}

Low ambient light is advisable in the reporting area and ambient lighting must not exceed 20 lux. $^{14}$ Following a reporting system such as the American College of Radiology (ACR) Breast Imaging-Reporting and Data System (BI-RADS) is advisable. ${ }^{15,16}$

\section{Breast Ultrasound}

\section{Introduction}

Breast ultrasound is a well-established and effective diagnostic modality for evaluation of breast diseases. The Indian radiologist is well versed with ultrasound as this is an easily available technique, both in large teaching hospitals as well as in small diagnostic centers across the country. Ultrasound breast is the primary imaging modality for younger women less than 30 years of age. It is an important adjunct tool to mammography especially for women with dense breasts. Technological advances and newer applications such as elastography and automated breast volume scanner (ABVS) have made ultrasound even more exciting and interesting. This document details the indications for breast ultrasound studies and also discusses the equipment, technique, and reporting of breast ultrasound examinations.

\section{Indication}

- Breast ultrasound is to be used as the initial imaging evaluation tool for palpable masses in younger women (younger than 30 years) at average risk of developing breast cancer. ${ }^{17}$ Based on the ultrasound findings and clinical features, mammography may be performed as required.

- Pregnant and lactating women: The absence of radiation makes ultrasound evaluation of breast symptoms in pregnant women a safe investigation. It is also an excellent tool for lactating women who are more likely to demonstrate dense breasts on mammography. Mastitis, breast abscess, and galactocele are the common pathologies seen in these women and these are very well demonstrated on ultrasound. ${ }^{18}$

- Nipple discharge: Evaluation of women with serous or sanguineous nipple discharge with high-resolution ultrasound gives direct visualization of dilated ducts and their contents. Color Doppler helps in differentiating between inspissated secretions and intraductal masses such as papillomas.

- Focal persistent pain: Focal pain in the breast which is noncyclical can be because of multiple reasons such as focal mastitis, fat necrosis, breast abscess, and hemorrhage in a cyst. Most of these conditions can be diagnosed and followed up on ultrasound. Diffuse bilateral cyclical breast pain does not warrant a breast ultrasound study.

- As an adjunct imaging modality: It is used as an adjunct imaging modality for further assessment of suspected or apparent abnormalities which are detected on mammography. These include abnormalities demonstrated on screening mammograms as well as diagnostic mammograms. Ultrasound is very useful to differentiate a palpable solid mass from a benign cyst. In malignant masses, it effectively demonstrates ductal extension of masses and is very useful to identify multifocal and multicentric disease in dense breasts.

- Second-look ultrasound after MRI: This allows identification of $\sim 68 \%$ of abnormalities seen only at MRI. ${ }^{19}$ Secondlook ultrasound is helpful in many situations. For example, screening MRI for women at high risk for breast cancer or preoperative breast MRI performed to assess multicentricity in known breast cancer patients may demonstrate MR abnormalities that were not demonstrated on mammography or ultrasound performed prior to breast MRI. Second-look ultrasound studies are highly recommended in such situations.

- Breast screening: Breast ultrasound is to be used as an adjunct to screening mammography for women with dense breasts and is deemed useful in the detection of mammographically occult cancers. Ultrasound replaces MRI for screening of women in the high-risk category if they are not suitable candidates for MRI or have no availability of MRI. ${ }^{4,17,20,21}$ However, ultrasound is not established as a primary screening modality for general populations and should not to be used as a standalone breast cancer screening tool.

- Follow-up of breast lesions: Sonographically well-visualized BI-RADS 3 lesions are best followed up in 6 months' time on ultrasound as there is no risk of radiation. However, if the lesion is not visualized on ultrasound, mammographic follow-up becomes necessary.

- Ultrasound-guided intervention: Ultrasound is the preferred imaging modality for image-guided procedures due to excellent real-time needle visualization, easy availability, patient comfort, and absence of radiation. Image-guided breast biopsy and other interventional procedures such as marker clip placement for patients treated with neoadjuvant chemotherapy and preoperative-guided hook wire 
localization of nonpalpable masses are all preferably performed under ultrasound guidance if the lesions are visualized sonographically. ${ }^{22,23}$ During treatment planning for radiation therapy, ultrasound is a valuable tool that helps assess large seromas, aspirate if clinically indicated and also is essential for ultrasound-guided boost irradiation of tumor cavity. ${ }^{24}$

- Imaging the axilla: Ultrasound is the modality of choice to image the axilla as well as to perform image-guided procedures in the axilla such as biopsy of axillary lymph nodes.

- Developing breast: Ultrasound is excellent for evaluation of developing breasts in young girls. Asymmetric breast development in this age group can be reassuringly differentiated from other pathologies by ultrasound.

- Male breast: Ultrasound evaluation of the male breast helps differentiate gynecomastia from breast masses such as breast carcinoma and breast abscess. It is also the modality of choice for guided procedures in the male breast.

- Augmented breast: It is the primary imaging modality for evaluation of breast implant-associated problems. Sonographic assessment of implant morphology, contour, content, and assessment of peri-implant tissues help evaluate implant-related complications such as infection, hematoma, capsular contracture, and rupture of implant.

- Investigation of an unknown primary: Ultrasound of the breast along with mammography is advised for investigation of patients presenting with metastases from an unknown primary.

\section{Equipment and Technical Factors}

Breast ultrasound should be performed with a real-time highresolution linear array transducer (such as $12-5 \mathrm{MHz}, 18-6$ $\mathrm{MHz}$ ) which has a center frequency of at least $10 \mathrm{MHz}$ and preferably higher. ${ }^{15,25}$ Characterization of breast lesions on ultrasound is highly dependent on technical factors. Depth, gain, and focal zone settings should be optimized for highquality images. The use of different modes and settings such as tissue harmonic imaging and compound imaging is helpful.

The patient should be positioned to minimize the thickness of the portion of the breast being evaluated. Ipsilateral arm should be up over/under the head. Image depth should be adjusted for complete visualization of breast tissue with chest wall on the posterior margin of the image. For evaluation of nipple areolar complex region and superficial lesions, the use of a thick layer of gel may be helpful.

\section{Ultrasound Evaluation and Documentation}

Breast ultrasound should be performed in correlation with patient symptoms, clinical signs, mammographic findings, and other breast imaging studies. For example, in a woman presenting with nipple discharge, detailed examination of the ducts must be meticulously performed. Following a reporting system such as the ACR BI-RADS is advisable. ${ }^{15}$

\section{Elastography}

Elastography is the sonographic method for imaging the elasticity of compliant tissues and provides information about stiffness of the lesion under evaluation. ${ }^{26}$ It is known that in general malignant breast masses tend to be harder due to their desmoplastic reaction when compared with the adjoining normal breast parenchyma and most benign lesions. The evaluation of breast masses to differentiate benign lesion from malignant is one of the most important applications of ultrasound elastography. ${ }^{26}$ It can help the radiologist to better characterize BI-RADS 3 and 4A masses, thus reducing unnecessary breast biopsies. ${ }^{27}$ However, elasticity of breast masses should only be used as an adjunct to B-mode ultrasound, not as a replacement for gray-scale ultrasound. ${ }^{10}$ In strain elastography, size ratio and strain ratio values should be documented. In shear wave, elastography elasticity value should be documented in kilopascals $(\mathrm{kPa})$ or meters/second $(\mathrm{m} / \mathrm{s}){ }^{27}$

\section{Automated Breast Volume Scanner}

ABVS acquires a whole series of consecutive B-mode images and reconstructs three-dimensional (3D) datasets of the entire breast volume. ABVS devices use mechanically driven wide linear array transducers that can image whole breast volumes in three dimensions. The data can be sent to a separate workstation to be independently analyzed by the radiologist. ${ }^{28}$ ABVS has potential advantages over conventional hand-held breast ultrasound as it is a standardized reproducible examination which gives dynamic cine loops of ultrasound images facilitating multiplanar and 3D reconstructions. ABVS is less operator dependent and it is being explored as a potential tool for breast cancer screening. ${ }^{29}$ Limited inclusion of the axillary tail and axilla and artifacts in the nipple area are some of the limitations of ABVS. Lesion detected on ABVS has to be further evaluated with hand-held ultrasound.

\section{MRI of the Breast}

\section{Introduction}

MRI of the breasts is an established, robust, and important imaging tool in the armamentarium of a trained breast radiologist for the detection and characterization of breast abnormalities. Its high sensitivity to detect breast cancer has led to MRI being established as an excellent screening tool in women with strong family history of breast cancer and with dense breasts and further for pretherapeutic local staging of newly diagnosed breast cancers where its role is being increasingly well accepted. It also serves as a good problem solving tool to clarify findings that are indeterminate on mammography and breast ultrasound.

Various groups and organizations have established recommendations for appropriate use of MRI, one such being the ACR which has laid down certain guidelines to standardize various aspects of conducting and reporting breast MRI studies in the ACR BI-RADS Atlas. At present, this is the most widely used MRI lexicon in India enabling clinicians across specialties to communicate well and work toward the common goal of better patient care. With increasing availability of breast MRI in facilities across our country, it is important to understand its advantages and limitations so that it can be utilized appropriately and effectively. 


\section{Prerequisites for Breast MRI}

- At least a 1.5-T magnet

- Dedicated bilateral breast surface coils capable of simultaneous bilateral imaging

- Equipment to perform mammographic correlation and directed breast ultrasonography

- Ideally MRI-guided intervention facility or at least a referral arrangement with a cooperating facility that could provide the service.

\section{Technique}

Although there may be minor variations in breast MRI acquisition protocols from center to center, there is a general agreement that high-quality imaging should include a technique that is bilateral, obtained using a dedicated breast coil with complete coverage of the breasts and axillae, is a dynamic multiphasic contrast-enhanced study, and has key pulse sequences with appropriate high spatial and temporal resolution for morphologic and kinetic assessment of the lesion. ${ }^{30}$

It is always a good idea to talk to the patient prior to the scan to obtain required history, clinically examine the patient, and to prepare her/him by explaining the entire procedure including the unusual prone position, contrast injection, and importance of not moving during scanning. Proper patient/breast positioning in the coil with application of optimal lateral compression plates to minimize movement and other inhomogeneous fat suppression artifacts balanced with adequate patient comfort is imperative in obtaining images of diagnostic quality.

Contrast agent and dose-Gadolinium contrast agent is injected intravenously at a dose of $0.1 \mathrm{mmol} / \mathrm{kg}$ followed by a 20 -mL saline flush at a rate of $\sim 2 \mathrm{~mL} / \mathrm{s}$, using a power injector. $^{30}$

Pulse sequences-For optimal diagnostic usefulness, a fluid-sensitive sequence with and without fat suppression (T2 FS/short tau inversion recovery), T1-weighted (T1W) and T2W two-dimensional or 3D images of at least $3 \mathrm{~mm}$ or less slice thickness with a maximum in-plane pixel dimension of $1 \mathrm{~mm}$ or less are required to achieve good spatial resolution. These are followed by a multiphase T1W dynamic contrastenhanced series with precontrast, initial postcontrast in a 60 - to 120 -second window for reasonable temporal resolution and subsequent delayed postcontrast images. ${ }^{30}$ Silicone selective sequences may be acquired for implant evaluation. Intravenous contrast administration can be omitted and a plain study performed for assessment of implant integrity. Newer techniques such as diffusion-weighted imaging and MR spectroscopy are optional.

Abbreviated (fast) breast MRI as a cost-effective screening protocol with similar sensitivity and specificity to a full diagnostic protocol may also be used with fewer sequences (3-4 in number) in varying combinations as per reader comfort to shorten the scan time. This would include a fluid sensitive T2/STIR, pre- and a single postcontrast fat suppressed T1W sequence.

Postprocessing techniques-Evaluation of images using subtraction, maximum intensity projections, morphologic and kinetic analysis on a dedicated workstation is essential.

\section{Indication}

Indications are divided into two main categories-screening and diagnostic.

\section{Screening Breast MRI}

Mammography is the investigation of choice for breast screening. However, mammography has its limitations especially in young high-risk women with dense breasts. Among other modalities, contrast-enhanced MRI has greater sensitivity compared with mammography and sonography for invasive (94$99 \%$ ) and in situ cancers (50-80\%) in high-risk population. Hence, MRI has been widely accepted in its role in the highrisk category of patients as an adjunct screening modality with mammography and not replacing it. It may also have a supplemental role in screening of the intermediate-risk category (15$20 \%$ ) in the future which is currently under research. ${ }^{31-34}$

Annual screening MRI along with mammogram should be offered in high-risk women, that is, those with a lifetime risk of breast cancer of $20 \%$ or more. ${ }^{31,33}$ This subset includes women with the following:

- Known BRCA1 or BRCA2 gene mutation

- Untested first-degree relative (mother, father, brother, sister, or child) of BRCA1 or BRCA2 gene mutation

- Those with a lifetime risk of breast cancer of 20 to $25 \%$ or more, according to risk assessment models

- Received mantle radiation to the chest for Hodgkin's disease between the ages of 10 and 30 years

- Having genetic disease such as Li-Fraumeni's syndrome, Cowden's syndrome, Bannayan-Riley-Ruvalcaba's syndrome, or first-degree relatives with these syndromes.

Breast MRI may also be considered as a supplement to mammography to screen women at intermediate risk of breast cancer (15-20\%) such as those with a personal history of breast cancer and dense tissue or for those diagnosed with breast cancer under the age of 50 years. ${ }^{35}$

Patients with breast augmentation-Screening breast MRI may also be considered in patients with silicone or saline implants and/or free injections with silicone, paraffin, or polyacrylamide gel in whom interpreting mammography may be difficult. It can also be considered for those who have undergone implant reconstruction following lumpectomy or mastectomy for breast cancer where contrast-enhanced breast MRI screening may be beneficial. ${ }^{35}$

\section{Diagnostic Breast MRI}

In its diagnostic role, breast MRI is helpful in the following clinical settings.

\section{Assessment of Extent of Disease in Newly Diagnosed Breast Cancer}

Although current literature does not support widespread use of MRI for breast cancer staging in terms of increasing overall survival and reducing re-excision rates, MRI does have the superior sensitivity and accuracy for detection of invasive and in situ disease as compared with CBE, mammography, and ultrasound with limited specificity and hence may be useful in selected subpopulations such as 
- In dense breasts to assess multifocality/multicentricity and ductal carcinoma in situ where it influences eligibility for breast conservation surgery (BCS)

- Lobular cancers which are more accurately imaged with MRI by virtue of their pattern of growth

- Posterior tumors better imaged with MRI for chest wall invasion

- Patients being planned for partial breast irradiation following BCS.

However, in view of its limited specificity, it is emphasized that all suspicious MR findings should be correlated with biopsy prior to definitive therapy to ensure appropriate treatment. Targeted second-look ultrasound, re-evaluation of mammograms, targeted mammographic views, or images obtained with digital breast tomosynthesis are useful, offering possibility of a biopsy under their guidance. Mass lesions identified on MRI are more likely to have a sonographic correlate than nonmasslike lesions (65 vs. $12 \%$, respectively). Hence, a second-look ultrasound is a useful diagnostic tool for lesions incidentally detected on breast MRI and also helps in guiding biopsies. In suspicious MR only detected lesions (BI-RADS 4 or 5), however, an MR-guided biopsy will be required. ${ }^{36}$

\section{Assessing Response to Neoadjuvant Chemotherapy}

In locally advanced breast cancers not amenable to upfront surgery, neoadjuvant chemotherapy is increasingly being used to reduce tumor size and enable BCS. In these cases MRI is very useful for evaluation of residual disease and to assess tumor responsiveness to chemotherapy.

\section{Metastatic Axillary Adenopathy with Occult Primary on} Clinical Breast Examination, Mammography, and Ultrasound MRI accurately detects the occult primary in 62 to $86 \%$ of cases $^{37}$ which is then treated accordingly. When MRI too is negative, axillary nodal dissection is done along with mastectomy or whole breast radiation therapy.

\section{Scar versus Tumor Recurrence}

Breast MRI is very useful when there is suspicion of disease recurrence in previously treated cases of breast cancer where the clinical and other conventional imaging modalities such as mammogram and ultrasound are equivocal, by virtue of the enhancement characteristics of tumor vis-à-vis the nonenhancing fibrotic scar. ${ }^{9}$

\section{For Problem Solving}

- In cases with equivocal or inconclusive findings on mammograms and ultrasound such as asymmetries with a suspicious appearance, multiple masses, pathological nipple discharge with no mammographic or sonographic correlate

- To localize lesions for image-guided biopsies and wire placements in cases where multiple solid lesions of similar characteristics are seen, to select the most suspicious ( 1 or 2 ) to biopsy
- Where the lesions are difficult to resolve sonographically such as intraductal inspissated secretions or intraductal solid lesion

- MRI only lesions can be picked up on relook ultrasound and hence helps in retrospective identification and ultrasound-guided biopsy of these lesions.

\section{Evaluation of Augmented Breasts}

MRI is the most sensitive imaging method to detect breast silicone implant integrity and does not require injection of intravenous contrast for assessing rupture only. Contrast may however be indicated in the evaluation of patients with silicone or saline implants and/or free injections with silicone, autologous fat, paraffin, or polyacrylamide gel, as well as for those who have undergone implant reconstruction following lumpectomy or mastectomy for breast cancer. The presence of implants does not affect the sensitivity of MRI for breast cancer detection.

\section{Reporting Breast MRI}

Report of MRI breasts should clearly mention the clinical indication for which it is being done along with findings noted on the other conventional imaging modalities, as it should never be interpreted in isolation. It is also important to remember that mammograms are obtained in upright position, ultrasound in supine/semilateral decubitus position, and MRI in prone position, which may lead to mild variation in the lesion location described as the o' clock position, especially those bordering the quadrants and should be noted as such. All findings need to be described as per standardized descriptors given in international guidelines such as the ACR BI-RADS system and a final impression should be mentioned indicating the worst comprehensive BIRADS category with a clear mention of further investigative advice such as biopsy or a follow up protocol. ${ }^{15}$

\section{Note}

The best practice guidelines of Breast Imaging Society, India (BISI) are the broad guidelines for investigation, intervention, and management of clients opting for breast screening and patients with breast symptoms in India, and intended for the use of qualified medical caregivers only. These are based on various national and international guidelines and personal experiences and opinions of BISI members, as there are no large credible Indian data to formulate these guidelines. These guidelines are purely recommendatory in nature. Actual decisions for management of patients should be individualized according to own judgment of the caregiver and tailored on case-to-case basis. As scientific knowledge is continuously improving, a regular update of the same by the caregiver is essential. Failure to do so may result in untoward patient management or outcome and BISI members or BISI as the organization cannot be held responsible for that in any manner.

Conflict of Interest

None declared. 


\section{References}

1 Pisano ED, Gatsonis C, Hendrick E, et al; Digital Mammographic Imaging Screening Trial (DMIST) Investigators Group. Diagnostic performance of digital versus film mammography for breast-cancer screening. N Engl J Med 2005;353(17): 1773-1783

2 National Centre for Diseases Informatics and Research. Government of India. Three year report of Population based Cancer Registries: 2012-2014;. National Cancer Registry Programme; Indian Council of Medical Research. Accessed July 2020: https:// ncdirindia.org/NCRP/ALL_NCRP_REPORTS/PBCR_REPORT_ 2012_2014/ALL_CONTENT/PDF_Printed_Version/Chapter10_ Printed.pdf

3 Public Health England. 2019). NHS Public Health Functions Agreement 2019-20; Service Specification no. 24, NHS Breast Screening Programme; Public Health England with NHS England and NHS Improvement and NHS Improvement Public Health Commissioning. Accessed September 2021: https://www.england.nhs.uk/wp-content/uploads/2017/04/Service-SpecificationNo.24-Breast_Screening_Programme.pdf

4 Lee CH, Dershaw DD, Kopans D, et al. Breast cancer screening with imaging: recommendations from the Society of Breast Imaging and the ACR on the use of mammography, breast MRI, breast ultrasound, and other technologies for the detection of clinically occult breast cancer. J Am Coll Radiol 2010;7(01):18-27

5 Brackstone M, Latosinsky S, Saettler E, George R. CJS debate: is mammography useful in average-risk screening for breast cancer? Can J Surg 2016;59(01):62-66

6 Guidance on Screening and Symptomatic Breast Imaging Fourth Edition, November 2019. Clinical Radiology, The Royal College of Radiologists. Accessed September 2021: https://www.rcr.ac.uk/system/files/publication/field_publication_files/bfcr199guidance-on-screening-and-symptomatic-breast-imaging.pdf

7 Magnus MC, Ping M, Shen MM, Bourgeois J, Magnus JH. Effectiveness of mammography screening in reducing breast cancer mortality in women aged 39-49 years: a meta-analysis. J Womens Health (Larchmt) 2011;20(06):845-852

8 Mainiero MB, Moy L, Baron P, et al; Expert Panel on Breast Imaging. ACR Appropriateness Criteria ${ }^{\circledR}$ breast cancer screening. J Am Coll Radiol 2017;14(11S):S383-S390

9 Agarwal G, Pradeep PV, Aggarwal V, Yip CH, Cheung PS. Spectrum of breast cancer in Asian women. World J Surg 2007;31(05):1031-1040

10 India Ministry of Home Affairs. 2019). Government of India. SRS Based Abridged Life Tables 2013-17, Office of the Registrar General \& Census Commissioner. Accessed September 2021: https://censusindia.gov.in/Vital_Statistics/SRS_Life_Table/SRS\% 20based\%20Abridged\%20Life\%20Tables\%202013-17.pdf

11 Holbrook AI, Moy L, Akin EA, et al; Expert Panel on Breast Imaging. ACR Appropriateness Criteria ${ }^{\circledR}$ breast pain. J Am Coll Radiol 2018; 15(1S):S276-S282

12 Salzman B, Fleegle S, Tully AS. Common breast problems. Am Fam Physician 2012;86(04):343-349

13 Department of Health. 2010). Government of UK. Best practice diagnostic guidelines for patients presenting with breast symptoms. Accessed September 2021: https://fdocuments.in/download/best-practice-diagnostic-guidelines-for-patients-presenting-with-breast-symptoms

14 Heggie JCP, Barnes P, Cartwright L, et al. Position paper: recommendations for a digital mammography quality assurance program V4.0. Australas Phys Eng Sci Med 2017;40(03):491-543

15 D'Orsi CJ, Sickles EA, Mendelson EB, et al. ACR BI-RADS ${ }^{\circledR}$ Atlas, Breast Imaging Reporting and Data System. Reston, VA: American College of Radiology; 2013

16 Breast Imaging Society. IndiaQuality Assurance Guidelines for Breast Imaging - Mammography. Accessed September 2021 at: https://www.bisi.co.in/mammography-qa-guidelines/
17 The American College of Radiology. ACR Practice parameter for the performance of a diagnostic breast ultrasound examination, Revised 2021 (Resolution 30). Accessed September 2021: https://www.acr. org/-/media/ACR/Files/Practice-Parameters/US-Breast.pdf

18 Mendelson EB. Problem-solving ultrasound. Radiol Clin North Am 2004;42(05):909-918, vii

19 Berg WA, Gutierrez L, NessAiver MS, et al. Diagnostic accuracy of mammography, clinical examination, US, and MR imaging in preoperative assessment of breast cancer. Radiology 2004;233 (03):830-849

20 Berg WA, Blume JD, Cormack JB, et al; ACRIN 6666 Investigators. Combined screening with ultrasound and mammography vs mammography alone in women at elevated risk of breast cancer. [published correction appears in JAMA 2010 Apr 21;303 (15):1482]JAMA 2008;299(18):2151-2163

21 Gordon PB. Ultrasound for breast cancer screening and staging. Radiol Clin North Am 2002;40(03):431-441

22 Parker SH, Jobe WE, Dennis MA, et al. US-guided automated largecore breast biopsy. Radiology 1993;187(02):507-511

23 The American College of Radiology. ACR Practice parameter for the performance of ultrasound-guided percutaneous breast interventional procedures, Revised 2021 (Resolution 31). Accessed September 2021: https://www.acr.org/-/media/ACR/Files/Practice-Parameters/us-guidedbreast.pdf

24 Farhan F, Esmati E, MaddahSafaei A, Shahriarian Sh, MiraiAshtiani MS, AkbariHamed E. Ultrasound-guided boost irradiation of tumor cavity after lumpectomy in breast cancer. Int J Radiat Res 2015;13(04):325-329

25 Canadian Association of Radiologists. CAR Practice guidelines and technical standards for Breast imaging and Intervention. Approved on September 29, 2012; modified on September 17, 2016. Accessed September 2021: https://car.ca/wp-content/uploads/ Breast-Imaging-and-Intervention-2016.pdf

26 Céspedes I, Ophir J, Ponnekanti H, Maklad N. Elastography: elasticity imaging using ultrasound with application to muscle and breast in vivo. Ultrason Imaging 1993;15(02):73-88

27 Youk JH, Gweon HM, Son EJ. Shear-wave elastography in breast ultrasonography: the state of the art. Ultrasonography 2017;36 (04):300-309

28 Wojcinski S, Farrokh A, Hille U, et al. The automated breast volume scanner (ABVS): initial experiences in lesion detection compared with conventional handheld B-mode ultrasound: a pilot study of 50 cases. Int J Womens Health 2011; 3:337-346

29 Kelly KM, Richwald GA. Automated whole-breast ultrasound: advancing the performance of breast cancer screening. Semin Ultrasound CT MR 2011;32(04):273-280

30 DeMartini WB, Rahbar H. Breast magnetic resonance imaging technique at $1.5 \mathrm{~T}$ and $3 \mathrm{~T}$ : requirements for quality imaging and American College of Radiology accreditation. Magn Reson Imaging Clin N Am 2013;21(03):475-482

31 The American College of Radiology. American College of Radiology ACR Appropriateness Criteria ${ }^{\circledR}$ breast cancer screening. Revised 2017. Accessed September 2021: https://acsearch.acr.org/docs/70910/narrative/

32 Boetes C. Update on screening breast MRI in high-risk women. Magn Reson Imaging Clin N Am 2010;18(02):241-247

33 Saslow D, Boetes C, Burke W, et al; American Cancer Society Breast Cancer Advisory Group. American Cancer Society guidelines for breast screening with MRI as an adjunct to mammography. [published correction appears in CA Cancer J Clin 2007 May-Jun;57(3):185]CA Cancer J Clin 2007;57(02): 75-89

34 Mahoney MC, Newell MS. Screening MR imaging versus screening ultrasound: pros and cons. Magn Reson Imaging Clin N Am 2013; 21(03):495-508 
68 Best Practice Guidelines for Breast Imaging Chakrabarthi et al.

35 The American College of Radiology. ACR practice parameter for the performance of contrast-enhanced magnetic resonance imaging (MRI) of the breast. Revised 2018 (Resolution 34). Accessed September 2021: https://www.acr.org/-/media/ACR/Files/Practice-Parameters/mr-contrast-breast.pdf
36 Brasic N, Wisner DJ, Joe BN. Breast MR imaging for extent of disease assessment in patients with newly diagnosed breast cancer. Magn Reson Imaging Clin N Am 2013;21(03):519-532

37 Argus A, Mahoney MC. Indications for breast MRI: case-based review. Am J Roentgenol 2011;196(3, Suppl):WS1-WS14 